Annals of Mathematics, 156 (2002), 931-946

\title{
Matzoh ball soup: Heat conductors with a stationary isothermic surface
}

\author{
By Rolando Magnanini and Shigeru Sakaguchi*
}

\begin{abstract}
We consider a bounded heat conductor that satisfies the exterior sphere condition. Suppose that, initially, the conductor has temperature 0 and, at all times, its boundary is kept at temperature 1. We show that if the conductor contains a proper sub-domain, satisfying the interior cone condition and having constant boundary temperature at each given time, then the conductor must be a ball.
\end{abstract}

\section{Introduction}

A matzoh ball is a dumpling, made of special unleavened crackers, that one takes from the refrigerator and drops into boiling stock (see [R-R] for a recipe). The physical situation at hand can be modeled in the general Euclidean space $\mathbb{R}^{N}$ as an initial-boundary value problem for the heat equation: in a bounded domain $\Omega$ - the matzoh ball - the normalized temperature $u=u(x, t)$ at a point $x \in \Omega$ and time $t>0$ satisfies the heat equation:

$$
u_{t}=\Delta u \text { in } \Omega \times(0,+\infty),
$$

and the two conditions:

$$
\begin{aligned}
& u=1 \text { on } \partial \Omega \times(0,+\infty), \\
& u=0 \text { on } \Omega \times\{0\} .
\end{aligned}
$$

A conjecture, posed in $[\mathrm{Kl}]$ by M. S. Klamkin and referred to by L. Zalcman in [Z] as the Matzoh Ball Soup, was settled affirmatively by G. Alessandrini in [A1]-[A2]. In [A2], under the assumption that every point of $\partial \Omega$ is regular

\footnotetext{
${ }^{*}$ This research was partially supported by a Grant-in-Aid for Scientific Research (C) ( $\left.¥ 10640175\right)$ and (B) ( $\sharp 12440042)$ of Japan Society for the Promotion of Science, by a 1999-2000 Grant of the Italian MURST and by the Visiting Professors program of INDAM-GNAFA-CNR.

Key words: Heat equation, overdetermined problems, stationary surfaces.

AMS subject classifications: Primary 35K05, 35K20, 35J05; Secondary 35J25, 35B38, 35B40.
} 
with respect to the Laplacian, it was proved that if all the spatial isothermic surfaces of $u$ are invariant with time then $\Omega$ must be a ball. (Of course, the values of $u$ vary with time on its spatial isothermic surfaces.)

The case where the homogeneous initial data in (1.3) is replaced by a function in the space $L^{2}(\Omega)$ was also considered in [A1]-[A2] and, with the help of J. Serrin's celebrated symmetry theorem for elliptic equations [Ser], was settled in the following terms: if all the spatial isothermic surfaces of the solution $u$ to (1.1) with homogeneous Dirichlet boundary condition and initial data $\varphi \in L^{2}(\Omega)$ are invariant with time, then either $\varphi$ is an eigenfunction of the Laplacian or $\Omega$ is a ball.

The analogous question where condition (1.2) is replaced by the homogeneous Neumann boundary condition was examined and answered positively (see [Sa1, Theorem 1]) with the aid of the classification theorem for isoparametric hypersurfaces in Euclidean space due to T. Levi-Civita and B. Segre (see [LC], [Seg]). The method used in [Sa1] can be applied to give an alternative proof of Alessandrini's results.

An important observation is that, in order to prove Klamkin's conjecture $[\mathrm{Kl}]$, both methods employed in [A1]-[A2] and [Sa1] need to assume that infinitely many isothermic surfaces of $u$ are invariant with time. As a natural consequence of this remark, one may wonder if the requirement that a finite number (possibly only one) of level surfaces of $u$ are invariant with time implies that $\Omega$ is a ball.

Our main result in this direction is the following.

THEOREM 1.1. Let $\Omega$ be a bounded domain in $\mathbb{R}^{N}, N \geq 2$, satisfying the exterior sphere condition and suppose that $D$ is a domain, with boundary $\partial D$, satisfying the interior cone condition, and such that $\bar{D} \subset \Omega$.

Assume that the solution $u$ to problem (1.1)-(1.3) satisfies the following condition:

$$
u(x, t)=a(t), \quad(x, t) \in \partial D \times(0,+\infty),
$$

for some function $a:(0,+\infty) \rightarrow(0,+\infty)$.

Then $\Omega$ must be a ball.

We recall that $\Omega$ satisfies the exterior sphere condition if for every $y \in \partial \Omega$ there exists a ball $B_{r}(z)$ such that $\overline{B_{r}(z)} \cap \bar{\Omega}=\{y\}$, where $B_{r}(z)$ denotes an open ball centered at $z \in \mathbb{R}^{N}$ and with radius $r>0$. Also, $D$ satisfies the interior cone condition if for every $x \in \partial D$ there exists a finite right spherical cone $K_{x}$ with vertex $x$ such that $K_{x} \subset \bar{D}$ and $\overline{K_{x}} \cap \partial D=\{x\}$.

When $\Omega$ is convex, we observe that there is no need to require that $D$ satisfies the interior cone condition. Indeed, a classical result shows that the function $x \mapsto \log (1-u(x, t))$ is concave for each given time $t>0$ (see [B-L], $[\mathrm{Ko}])$. This fact and the analyticity of $u$ in $x$, imply that, for each $t>0$, there 
exists a point $x(t) \in \Omega$ - the cold spot - such that

$$
\{x \in \Omega: \nabla u(x, t)=0\}=\left\{x \in \Omega: u(x, t)=\min _{y \in \Omega} u(y, t)\right\}=\{x(t)\} .
$$

Thus, we can conclude that, with the exception of the cold spot and the boundary $\partial \Omega$, the isothermic surfaces in a convex conductor are always smooth closed convex hypersurfaces. The following result is then an easy consequence of Theorem 1.1.

Corollary 1.2. Let $\Omega$ be a bounded convex domain in $\mathbb{R}^{N}, N \geq 2$, and suppose that $D$ is a domain such that $\bar{D} \subset \Omega$. Assume that the solution $u$ to problem (1.1)-(1.3) satisfies condition (1.4).

Then $\Omega$ must be a ball.

The proof of Theorem 1.1 exploits arguments different from the ones used in [A1]-[A2] and [Sa1]. Our technique is essentially based on two ingredients.

One ingredient is a careful study of the asymptotic behavior of $u(x, t)$ as $t \rightarrow 0^{+}$or, more conveniently, the asymptotic behavior as $s \rightarrow+\infty$ of the function $W=W(x, s)$ defined by

$$
W(x, s)=s \int_{0}^{+\infty} u(x, t) e^{-s t} d t, s>0 .
$$

Notice that $W$ is the solution of the following elliptic boundary value problem:

$$
\begin{array}{ll}
\Delta W-s W=0 & \text { in } \Omega, \\
W=1 & \text { on } \partial \Omega .
\end{array}
$$

A result in [Va] (see also [F-W] and [E-I]) shows that, as $s \rightarrow+\infty$, the function $-\frac{1}{\sqrt{s}} \log W(x, s)$ converges uniformly on $\bar{\Omega}$ to the function $d=d(x)$ defined by

$$
d(x)=\operatorname{dist}(x, \partial \Omega), \quad x \in \Omega .
$$

Moreover, if $u$ satisfies (1.4), then for any fixed $s>0, W$ is constant on $\partial D$ : indeed,

$$
W(x, s)=s \int_{0}^{+\infty} a(t) e^{-s t} d t:=A(s), x \in \partial D .
$$

In Section 3, by using these observations, we will show two facts:

(i) $\Omega=D+B_{R}(0)$, where $B_{R}(0)$ is the ball centered at the origin and with radius

$$
R=\lim _{s \rightarrow+\infty}\left\{-\frac{1}{\sqrt{s}} \log A(s)\right\} ;
$$

in other words $\partial \Omega$ and $\partial D$ are parallel surfaces; 
(ii) $\partial D$ is analytic and, since $\partial D$ is a level surface of $d$, also $\partial \Omega$ must be real analytic.

The second ingredient of our proof is the balance law proved in Theorem 2.1. Let $G$ be a domain in $\mathbb{R}^{N}$; a solution $v=v(x, t)$ to the heat equation in $G \times(0,+\infty)$ is such that $v\left(x_{0}, t\right)=0$, for some $x_{0} \in G$ and for every $t>0$, if and only if

$$
\int_{\partial B_{r}\left(x_{0}\right)} v(x, t) d S_{x}=0, \text { for every } r \in\left[0, d_{*}\right) \text { and every } t>0,
$$

where $d_{*}=\operatorname{dist}\left(x_{0}, \partial G\right)$. If $v$ is bounded, we introduce a function $V=V(x, s)$ defined as in (1.5) by replacing $u$ with $v$ and we derive from (1.11) that

$$
\int_{\partial B_{r}\left(x_{0}\right)} V(x, s) d S_{x}=0, \text { for every } r \in\left[0, d_{*}\right) \text { and every } s>0 .
$$

By (1.12) and the study of the asymptotic behavior of the integral in (1.12) as $s \rightarrow+\infty$ (see Theorem 2.3) we will show in Theorem 3.2 that if the solution $u$ to (1.1)-(1.3) satisfies (1.4) then

$$
\prod_{j=1}^{N-1}\left[\frac{1}{R}-\kappa_{j}(x)\right]=\text { constant, for every } x \in \partial \Omega .
$$

Here, $\kappa_{j}(x), j=1, \ldots, N-1$, denotes the $j^{\text {th }}$ principal curvature of the surface $\partial \Omega$ at the point $x \in \partial \Omega$ (we refer to $\S 2$ for a definition of $\kappa_{j}$ ).

If $N=2$, condition (1.13) directly implies that $\Omega$ is a ball. When $N \geq 3$, we derive the same conclusion with the help of A. D. Aleksandrov's uniqueness theorem [Alek].

Theorem 2.1 was stated without proof in [M-S2]. To make the present paper self-contained, we present a short proof of Theorem 2.1 together with a new proof of a result (Corollary 2.2) proved in [M-S1]. A more general version of Theorem 2.3 will appear in a forthcoming paper ([M-S3]).

\section{A balance law and an asymptotic estimate}

In this section, we shall construct the two main tools for proving our symmetry results. One of them is the following balance law.

TheOREM 2.1. Let $G$ be a domain in $\mathbb{R}^{N}, N \geq 2$, let $x_{0}$ be a point in $G$ and set $d_{*}=\operatorname{dist}\left(x_{0}, \partial G\right)$. Suppose that $v=v(x, t)$ is a solution of the heat equation in $G \times(0,+\infty)$.

Then the following assertions are equivalent:

(i) $v\left(x_{0}, t\right)=0$ for every $t \in(0,+\infty)$; 
(ii) for every $(r, t) \in\left[0, d_{*}\right) \times(0,+\infty)$

$$
\int_{\partial B_{r}\left(x_{0}\right)} v(x, t) d S_{x}=0 .
$$

Proof. By a translation, we can suppose that $x_{0}=0$, the origin of $\mathbb{R}^{N}$. If $(2.1)$ holds, then $v(0, t)=0$ for every $t \in(0,+\infty)$ clearly. If $v(0, t)=0$ for every $t \in(0,+\infty)$, we will show that the function $p=p(r, t)$ defined in $\left[0, d_{*}\right) \times(0,+\infty)$ by

$$
p(r, t)=\int_{\partial B_{1}(0)} v(r x, t) d S_{x},
$$

which is analytic with respect to $r$ in $\left[0, d_{*}\right)$, is a solution of the initial value problem:

$$
\begin{aligned}
& p_{t}=p_{r r}+\frac{N-1}{r} p_{r} \text { in }\left(0, d_{*}\right) \times(0,+\infty), \\
& p(0, t)=p_{r}(0, t)=0, \quad t \in(0,+\infty) .
\end{aligned}
$$

Hence, (2.1) follows from the fact that

$$
\frac{\partial^{k} p}{\partial r^{k}}(0, t)=0, t \in(0,+\infty), k=0,1,2, \ldots,
$$

by induction on the integer $k$ (see [Sa2] for a similar argument).

It is evident that $p(0, t)=0$ for every $t \in(0,+\infty)$. As in [M-S1], by using the heat equation in radial coordinates, we write

$$
0=\int_{\partial B_{1}(0)}\left(\partial_{t}-\partial_{r}^{2}-\frac{N-1}{r} \partial_{r}-\frac{1}{r^{2}} \Delta_{\mathbb{S}^{N-1}}\right) v(r x, t) d S_{x},
$$

where $\Delta_{\mathbb{S}^{N-1}}$ denotes the Laplace-Beltrami operator on $\mathbb{S}^{N-1} \equiv \partial B_{1}(0)$. Then (2.3) follows from the fact that $\int_{\partial B_{1}(0)} \Delta_{\mathbb{S}^{N-1}} v(r x, t) d S_{x}=0$.

Finally, from (2.3), we have

$$
p_{r}(0, t)=\frac{1}{N-1} \lim _{r \rightarrow 0} r\left(p_{t}-p_{r r}\right)=0
$$

for every $t \in(0,+\infty)$.

The following corollary provides another proof of a result first demonstrated in [M-S1].

Corollary 2.2. Assume $G, x_{0}, d_{*}$ and $v$ as in Theorem 2.1. Then the following assertions are equivalent:

(i) $\nabla v\left(x_{0}, t\right)=0$ for every $t \in(0,+\infty)$; 
(ii) for every $(r, t) \in\left[0, d_{*}\right) \times(0,+\infty)$

$$
\int_{\partial B_{r}\left(x_{0}\right)}\left(x-x_{0}\right) v(x, t) d S_{x}=0 .
$$

Proof. Since each component of $\nabla v(x, t)$ satisfies the heat equation, by Theorem 2.1 we have that (i) is equivalent to

$$
\int_{\partial B_{r}\left(x_{0}\right)} \nabla v(x, t) d S_{x}=0 \text { for every }(r, t) \in\left[0, d_{*}\right) \times(0,+\infty) .
$$

Integrating the latter formula with respect to $r$ yields

$$
\int_{B_{r}\left(x_{0}\right)} \nabla v(x, t) d x=0 \text { for every }(r, t) \in\left[0, d_{*}\right) \times(0,+\infty),
$$

which, by the divergence theorem, is equivalent to (2.5).

Theorem 2.3 below provides our second tool for the proofs of our symmetry results. In order to state it, we need to introduce some notation and definitions.

Take a point $x \in \partial \Omega$ and a unit vector $\omega \in T_{x}(\partial \Omega)$ - the tangent space to $\partial \Omega$ at $x$ - and let $\sigma \mapsto \gamma(\sigma)$ be a smooth curve on $\partial \Omega$, parametrized according to its arclength $\sigma \in[0, L]$, such that $\gamma(0)=x$ and $\gamma^{\prime}(0)=\omega$.

Define a function $\mathcal{S}_{x}:\left\{\omega \in T_{x}(\partial \Omega):|\omega|=1\right\} \rightarrow \mathbb{R}$ by

$$
\mathcal{S}_{x}(\omega)=\gamma^{\prime \prime}(0) \cdot \nu(x)
$$

where $\nu(x)$ is the interior unit normal vector to $\partial \Omega$ at $x$ and the dot denotes scalar product. Notice that $\mathcal{S}_{x}(\omega)$ is the curvature of the curve $\gamma$ at $x$, by the Frenet-Serret formulae.

Let $d(x)$ be defined by (1.8); since $\nu(x)=\nabla d(x)$ and $\gamma^{\prime}(\sigma) \cdot \nu(\gamma(\sigma))=0$ for every $\sigma \in[0, L]$, by differentiating with respect to $\sigma$ this latter equation, we obtain:

$$
\gamma^{\prime \prime}(\sigma) \cdot \nu(\gamma(\sigma))=-\gamma^{\prime}(\sigma) \cdot\left[\nabla^{2} d(\gamma(\sigma)) \gamma^{\prime}(\sigma)\right],
$$

where $\nabla^{2} d$ denotes the Hessian matrix of $d$, and hence

$$
\mathcal{S}_{x}(\omega)=-\omega \cdot\left[\nabla^{2} d(x) \omega\right], \omega \in T_{x}(\partial \Omega) \text { with }|\omega|=1 .
$$

We can extend $\mathcal{S}_{x}$ to a bilinear form - the shape operator at $x$ - on $\mathbb{R}^{N}=$ $T_{x}\left(\mathbb{R}^{N}\right)$ by observing that $\omega \cdot\left[\nabla^{2} d(x) \omega\right]=0$ for every $\omega$ proportional to $\nu(x)=\nabla d(x)$; in fact, $\nabla^{2} d(x) \nabla d(x)=0$, since $|\nabla d|^{2}=1$ on $\Omega$ (see [G-H-L]). The critical values of $\mathcal{S}_{x}(\omega)$ on the unit sphere $\mathbb{S}^{N-1}$ - the eigenvalues of $-\nabla^{2} d(x)$ - are 0 and the principal curvatures $\kappa_{1}(x), \ldots, \kappa_{N-1}(x)$ of $\partial \Omega$ at $x$ (see [G-T, Lemma 14.17]). 
Theorem 2.3. Let $\Omega \subset \mathbb{R}^{N}$ be a domain with $C^{2}$ boundary $\partial \Omega$ and let $\kappa_{1}, \ldots, \kappa_{N-1}$ denote the principal curvatures of $\partial \Omega$.

Let $B_{R}\left(x_{0}\right) \subset \Omega$ be an open ball with radius $R>0$ centered at $x_{0}$ and suppose that the set $\partial \Omega \cap \partial B_{R}\left(x_{0}\right)$ is made of a finite number of points $p_{1}, \ldots, p_{K}$ such that $\kappa_{j}\left(p_{k}\right)<\frac{1}{R}$ for every $j=1, \ldots, N-1$ and every $k=1, \ldots, K$.

Let $W=W(x, s)$ be the solution to problem (1.6)-(1.7). Then, the following formula holds for every function $\varphi$ continuous on $\mathbb{R}^{N}$ :

$$
\begin{aligned}
& \lim _{s \rightarrow+\infty} s^{\frac{N-1}{4}} \int_{\partial B_{R}\left(x_{0}\right)} \varphi(x) W(x, s) d S_{x} \\
& =(2 \pi)^{\frac{N-1}{2}} \sum_{k=1}^{K} \varphi\left(p_{k}\right)\left\{\prod_{j=1}^{N-1}\left[\frac{1}{R}-\kappa_{j}\left(p_{k}\right)\right]\right\}^{-\frac{1}{2}} .
\end{aligned}
$$

The proof of Theorem 2.3 is based on Lemma 2.4 below, where we show that the two functions

$$
W_{\varepsilon}^{ \pm}(x, s)=\exp \{-\sqrt{s(1 \mp \varepsilon)} d(x)\},
$$

where $d(x)$ is defined by (1.8), provide respectively an upper and a lower barrier for $W$ in $\Omega$ for large values of $s$.

Lemma 2.4. Let $\Omega$ be a bounded domain in $\mathbb{R}^{N}$ with $C^{2}$ boundary $\partial \Omega$. Let $W(x, s)$ be the solution to (1.6)-(1.7).

Then, for every $\varepsilon>0$, there exists a positive number $s_{\varepsilon}$ such that

$$
W_{\varepsilon}^{-}(x, s) \leq W(x, s) \leq W_{\varepsilon}^{+}(x, s)
$$

for every $x \in \bar{\Omega}$ and every $s \geq s_{\varepsilon}$, where $W_{\varepsilon}^{-}(x, s)$ and $W_{\varepsilon}^{+}(x, s)$ are defined in (2.8).

Proof. Choose a number $\delta>0$ such that the function $d=d(x)$ defined in (1.8) is of class $C^{2}$ in the set $\overline{\Omega_{\delta}}$ where

$$
\Omega_{\delta}=\{x \in \Omega: d(x)<\delta\} .
$$

Let $W_{\varepsilon}^{ \pm}(x, s)$ be given by (2.8). A straightforward computation gives

$$
\Delta W_{\varepsilon}^{ \pm}-s W_{\varepsilon}^{ \pm}=\mp \varepsilon \sqrt{s}\left\{\sqrt{s} \pm \frac{\sqrt{(1 \mp \varepsilon)}}{\varepsilon} \Delta d\right\} W_{\varepsilon}^{ \pm} \quad \text { in } \Omega_{\delta} .
$$

Set $M_{\delta}=\max _{\bar{\Omega}_{\delta}}|\Delta d| ;$ if $s \geq \frac{1+\varepsilon}{\varepsilon^{2}} M_{\delta}^{2}$, then

$$
\begin{aligned}
& \Delta W_{\varepsilon}^{+}-s W_{\varepsilon}^{+} \leq 0 \\
& \Delta W_{\varepsilon}^{-}-s W_{\varepsilon}^{-} \geq 0
\end{aligned} \quad \text { in } \quad \Omega_{\delta} .
$$


Since the function $-\frac{1}{\sqrt{s}} \log W(x, s)$ converges uniformly on $\bar{\Omega}$ to $d(x)$ as $s \rightarrow+\infty$, (see $[\mathrm{Va}],[\mathrm{E}-\mathrm{I}]$ ), there exists a number $s^{*}>0$ such that

$$
-\delta(1-\sqrt{1-\varepsilon}) \leq-\frac{1}{\sqrt{s}} \log W(x, s)-d(x) \leq \delta(\sqrt{1+\varepsilon}-1), x \in \bar{\Omega},
$$

for every $s \geq s^{*}$. Hence, since $d(x) \geq \delta$ for every $x \in \Omega \backslash \Omega_{\delta}$, we obtain

$$
W_{\varepsilon}^{-}(x, s) \leq W(x, s) \leq W_{\varepsilon}^{+}(x, s), \quad x \in \Omega \backslash \Omega_{\delta},
$$

for every $s \geq s^{*}$. Moreover,

$$
W_{\varepsilon}^{-}(x, s)=W(x, s)=W_{\varepsilon}^{+}(x, s)=1, \quad x \in \partial \Omega,
$$

for every $s>0$, clearly.

Choose $s_{\varepsilon}=\max \left(s^{*}, \frac{1+\varepsilon}{\varepsilon^{2}} M_{\delta}^{2}\right)$. Then by the comparison principle, from (2.11), (2.12) and (2.13), we have

$$
W_{\varepsilon}^{-}(x, s) \leq W(x, s) \leq W_{\varepsilon}^{+}(x, s), \quad x \in \Omega_{\delta},
$$

for every $s \geq s_{\varepsilon}$. Combining (2.14) with (2.12) yields (2.9).

Proof of Theorem 2.3. We will show preliminarily that

$$
\begin{aligned}
\lim _{s \rightarrow+\infty} s^{\frac{N-1}{4}} & \int_{\partial B_{R}\left(x_{0}\right)} \varphi(x) e^{-\sqrt{s} d(x)} d S_{x} \\
= & (2 \pi)^{\frac{N-1}{2}} \sum_{k=1}^{K} \varphi\left(p_{k}\right)\left\{\prod_{j=1}^{N-1}\left[\frac{1}{R}-\kappa_{j}\left(p_{k}\right)\right]\right\}^{-\frac{1}{2}} .
\end{aligned}
$$

Let $p_{h} \in\left\{p_{1}, \ldots, p_{K}\right\}$; by using a partition of unity, we can suppose that $\operatorname{supp} \varphi$ does not contain the point $2 x_{0}-p_{h}$ and any $p_{k}$ different from $p_{h}$.

Let $\mathbb{R}^{N-1} \ni \sigma=\left(\sigma_{1}, \ldots, \sigma_{N-1}\right) \mapsto x(\sigma) \in \partial B_{R}\left(x_{0}\right)$ be a parametrization of $\partial B_{R}\left(x_{0}\right)$ such that $x(0)=p_{h}$; a convenient choice of $x(\sigma)$ is the stereographic projection from the point $2 x_{0}-p_{h}$ onto the tangent space to $\partial B_{R}\left(x_{0}\right)$ at $p_{h}$. Precisely, take an orthonormal basis $\xi^{1}, \ldots, \xi^{N}$ of $\mathbb{R}^{N}$ with $\xi^{N}=\left(x_{0}-p_{h}\right) / R$, and put:

$$
x(\sigma)=\frac{2 R|\sigma|^{2}}{4 R^{2}+|\sigma|^{2}} \xi^{N}+\frac{4 R^{2}}{4 R^{2}+|\sigma|^{2}} \sum_{j=1}^{N-1} \sigma_{j} \xi^{j}+p_{h} .
$$

By this change of coordinates, the integral in (2.15) becomes

$$
\int_{\partial B_{R}\left(x_{0}\right)} \varphi(x) e^{-\sqrt{s} d(x)} d S_{x}=\int_{\mathbb{R}^{N-1}} \varphi(x(\sigma)) e^{-\sqrt{s} d(x(\sigma))} J(\sigma) d \sigma,
$$

where

$$
J(\sigma) \equiv \sqrt{\operatorname{det}\left(\frac{\partial x(\sigma)}{\partial \sigma_{i}} \cdot \frac{\partial x(\sigma)}{\partial \sigma_{j}}\right)}=\left(\frac{4 R^{2}}{4 R^{2}+|\sigma|^{2}}\right)^{N-1},
$$


since

$$
\frac{\partial x(\sigma)}{\partial \sigma_{i}} \cdot \frac{\partial x(\sigma)}{\partial \sigma_{j}}=\left(\frac{4 R^{2}}{4 R^{2}+|\sigma|^{2}}\right)^{2} \delta_{i j}, i, j=1, \ldots, N-1 .
$$

Here $\delta_{i j}$ is Kronecker's symbol.

Let $d^{*}(\sigma)=d(x(\sigma))$. Then $d^{*}(0)=0$. We will later observe that $\nabla d^{*}(0)=0$ and $\nabla^{2} d^{*}(0)$ is positive definite. Moreover, since $\operatorname{supp} \varphi$ does not contain any $p_{k}$ different from $p_{h}$, we may assume that $d^{*}(\sigma)>0$ if $\sigma \neq 0$. Hence, by Laplace's method (see [deB, p. 71] for example), or by the stationary phase method (see [Ev, pp. 208-217] for example), we infer that

$$
\begin{gathered}
\lim _{s \rightarrow+\infty} s^{\frac{N-1}{4}} \int_{\mathbb{R}^{N-1}} \varphi(x(\sigma)) e^{-\sqrt{s} d(x(\sigma))} J(\sigma) d \sigma \\
=(2 \pi)^{\frac{N-1}{2}} \varphi\left(p_{h}\right) J(0)\left(\operatorname{det} \nabla^{2} d^{*}(0)\right)^{-\frac{1}{2}} .
\end{gathered}
$$

Formula (2.15) will result from (2.17) by observing that $J(0)=1$ and that

$$
\operatorname{det} \nabla^{2} d^{*}(0)=\prod_{j=1}^{N-1}\left[\frac{1}{R}-\kappa_{j}\left(p_{h}\right)\right]
$$

as it will be clear from the following argument.

Differentiating $d^{*}(\sigma)$ twice yields:

$$
\begin{aligned}
\frac{\partial^{2} d^{*}}{\partial \sigma_{i} \partial \sigma_{j}}(\sigma)= & \frac{\partial x}{\partial \sigma_{i}}(\sigma) \cdot\left(\nabla^{2} d(x(\sigma)) \frac{\partial x}{\partial \sigma_{j}}(\sigma)\right) \\
& +\nabla d(x(\sigma)) \cdot \frac{\partial^{2} x}{\partial \sigma_{i} \partial \sigma_{j}}(\sigma), \quad i, j=1, \ldots, N-1,
\end{aligned}
$$

for every $\sigma \in \mathbb{R}^{N-1}$, where the dot stands for scalar product of vectors in $\mathbb{R}^{N}$.

Since $x(\sigma) \in \partial B_{R}\left(x_{0}\right)$ for every $\sigma \in \mathbb{R}^{N-1}$, we obtain:

$$
\begin{aligned}
& \frac{\partial x}{\partial \sigma_{i}}(\sigma) \cdot\left(x(\sigma)-x_{0}\right)=0, \quad i=1, \ldots, N-1, \\
& \frac{\partial^{2} x}{\partial \sigma_{i} \partial \sigma_{j}}(\sigma) \cdot\left(x(\sigma)-x_{0}\right)+\frac{\partial x}{\partial \sigma_{i}}(\sigma) \cdot \frac{\partial x}{\partial \sigma_{j}}(\sigma)=0, \quad i, j=1, \ldots, N-1,
\end{aligned}
$$

for every $\sigma \in \mathbb{R}^{N-1}$. The fact that $-\nabla d\left(p_{h}\right)=\left(x(0)-x_{0}\right) / R$ then yields that

$$
\begin{aligned}
& \nabla d\left(p_{h}\right) \cdot \frac{\partial x}{\partial \sigma_{i}}(0)=0, \quad i=1, \ldots, N-1, \\
& \nabla d\left(p_{h}\right) \cdot \frac{\partial^{2} x}{\partial \sigma_{i} \partial \sigma_{j}}(0)=\frac{1}{R} \frac{\partial x}{\partial \sigma_{i}}(0) \cdot \frac{\partial x}{\partial \sigma_{j}}(0), \quad i, j=1, \ldots, N-1,
\end{aligned}
$$


and hence

$$
\begin{aligned}
\nabla d^{*}(0) & =0 \\
\frac{\partial^{2} d^{*}}{\partial \sigma_{i} \partial \sigma_{j}}(0) & =\frac{\partial x}{\partial \sigma_{i}}(0) \cdot\left\{\left[\nabla^{2} d\left(p_{h}\right)+\frac{1}{R} I\right] \frac{\partial x}{\partial \sigma_{j}}(0)\right\}, i, j=1, \ldots, N-1,
\end{aligned}
$$

by (2.19), where $I$ is the $N \times N$ identity matrix.

By (2.16), the vectors $\frac{\partial x}{\partial \sigma_{i}}(0), i=1, \ldots, N-1$, make an orthonormal basis of the tangent space $T_{p_{h}}(\partial \Omega)=T_{p_{h}}\left(\partial B_{R}\left(x_{0}\right)\right)$; therefore, we conclude that the eigenvalues of $\nabla^{2} d^{*}(0)$ are $\frac{1}{R}-\kappa_{j}\left(p_{h}\right)(j=1, \ldots, N-1)$ and hence $(2.18)$ holds.

We now prove formula (2.7). It suffices to prove it for any nonnegative $\varphi$, since any $\varphi$ can be written as $\varphi=\varphi^{+}-\varphi^{-}$where $\varphi^{+}=\max \{\varphi, 0\}$ and $\varphi^{-}=\max \{-\varphi, 0\}$.

By Lemma 2.4, we have for every $s \geq s_{\varepsilon}$ and for every nonnegative $\varphi$ :

$\int_{\partial B_{R}\left(x_{0}\right)} \varphi(x) W_{\varepsilon}^{-}(x, s) d S_{x} \leq \int_{\partial B_{R}\left(x_{0}\right)} \varphi(x) W(x, s) d S_{x} \leq \int_{\partial B_{R}\left(x_{0}\right)} \varphi(x) W_{\varepsilon}^{+}(x, s) d S_{x} ;$

then, (2.15) and the definition $(2.8)$ of $W_{\varepsilon}^{ \pm}(x, s)$ give

$$
\begin{aligned}
& \limsup _{s \rightarrow+\infty} s^{\frac{N-1}{4}} \int_{\partial B_{R}\left(x_{0}\right)} \varphi(x) W(x, s) d S_{x} \\
& \leq\left(\frac{2 \pi}{\sqrt{1-\varepsilon}}\right)^{\frac{N-1}{2}} \sum_{k=1}^{K} \varphi\left(p_{k}\right)\left\{\prod_{j=1}^{N-1}\left[\frac{1}{R}-\kappa_{j}\left(p_{k}\right)\right]\right\}^{-\frac{1}{2}}
\end{aligned}
$$

and

$$
\begin{aligned}
\liminf _{s \rightarrow+\infty} s^{\frac{N-1}{4}} \int_{\partial B_{R}\left(x_{0}\right)} \varphi(x) W(x, s) d S_{x} \\
\geq\left(\frac{2 \pi}{\sqrt{1+\varepsilon}}\right)^{\frac{N-1}{2}} \sum_{k=1}^{K} \varphi\left(p_{k}\right)\left\{\prod_{j=1}^{N-1}\left[\frac{1}{R}-\kappa_{j}\left(p_{k}\right)\right]\right\}^{-\frac{1}{2}},
\end{aligned}
$$

for every $\varepsilon>0$. By letting $\varepsilon$ tend to 0 , we obtain (2.7) and the proof is concluded.

\section{Symmetry results}

In Lemma 3.1 below, we prove analyticity of $\partial D$ and $\partial \Omega$ by using our balance law. 
Lemma 3.1. Let $\Omega$ be a bounded domain in $\mathbb{R}^{N}, N \geq 2$, satisfying the exterior sphere condition and suppose that $D$ is a domain satisfying the interior cone condition and such that $\bar{D} \subset \Omega$.

Assume that the solution $u=u(x, t)$ to problem (1.1)-(1.3) satisfies condition (1.4). Let $R$ be the positive constant given by (1.10).

Then the following assertions hold:

(i) for every $x \in \partial D, d(x)=R$, where $d$ is defined by (1.8);

(ii) $\partial D$ is analytic;

(iii) $\partial \Omega$ is analytic and $\partial \Omega=\left\{x \in \mathbb{R}^{N}: \operatorname{dist}(x, D)=R\right\}$;

(iv) the mapping: $\partial D \ni x \mapsto y(x) \equiv x-R \nu^{*}(x) \in \partial \Omega$ is a diffeomorphism, where $\nu^{*}(x)$ denotes the interior unit normal vector to $\partial D$ at $x \in \partial D$;

(v) for every $x \in \partial D, \nabla d(y(x))=\nu^{*}(x)$ and $\overline{B_{R}(x)} \cap \partial \Omega=\{y(x)\}$;

(vi) let $\kappa_{j}(y), j=1, \ldots, N-1$ denote the $j^{\text {th }}$ principal curvature at $y \in \partial \Omega$ of the analytic surface $\partial \Omega$; then $\kappa_{j}(y)<\frac{1}{R}, j=1, \ldots, N-1$, for every $y \in \partial \Omega$.

Proof. (i) As already observed, under our assumptions, for each fixed $s>0$, the function $W=W(x, s)$, defined by (1.5), is the solution to problem (1.6)-(1.7) and satisfies (1.9). Since $\Omega$ enjoys the exterior sphere condition, we can apply a result in [Va] (see also [E-I] and [F-W]): as $s \rightarrow+\infty$, the function $-\frac{1}{\sqrt{s}} \log W(x, s)$ converges uniformly on $\bar{\Omega}$ to the function $d(x)$ defined by (1.8), and hence we get (i).

(ii) It suffices to show that, for every point $x \in \partial D$, there exists a time $t^{*}>0$ such that $\nabla u\left(x, t^{*}\right) \neq 0$; then, analyticity of $\partial D$ will follow from analyticity of $u$ with respect to the space variable.

Assume by contradiction that there exists a point $x_{0} \in \partial D$ such that $\nabla u\left(x_{0}, t\right)=0$ for every $t>0$. Since $u$ is continuous up to $\partial \Omega \times(0,+\infty)$, by Corollary 2.2 (ii), we can infer that

$$
\int_{\partial B_{R}\left(x_{0}\right)}\left(x-x_{0}\right) u(x, t) d S_{x}=0 \text { for every } t>0
$$

hence

$$
\int_{\partial B_{R}\left(x_{0}\right)}\left(x-x_{0}\right) W(x, s) d S_{x}=0 \text { for every } s>0,
$$

in view of (1.5).

On the other hand, since $D$ satisfies the interior cone condition, there exists a finite right spherical cone $K$ with vertex at $x_{0}$ such that $K \subset \bar{D}$ and 
$\bar{K} \cap \partial D=\left\{x_{0}\right\}$. By translating and rotating if needed, we can suppose that $x_{0}=0$ and that $K$ is the set $\left\{x \in B_{\rho}(0): x_{N}<-|x| \cos \theta\right\}$, where $\rho \in(0, R)$ and $\theta \in\left(0, \frac{\pi}{2}\right)$.

Since $K \subset \bar{D}$ and $\bar{K} \cap \partial D=\{0\}$, assertion (i) implies that

$$
d(x)>R \text { for every } x \in K \text {. }
$$

The set defined by

$$
V=\left\{x \in \partial B_{R}(0): x_{N} \geq R \sin \theta\right\}
$$

is such that

$$
\partial \Omega \cap \partial B_{R}(0) \subset V,
$$

because, otherwise, there would be a point in $K$ contradicting (3.2).

Thus, from (3.4) it follows that we can choose a number $\delta>0$ such that

$$
d(x) \geq 5 \delta \text { for every } x \in \partial B_{R}(0) \cap\left\{x_{N} \leq 0\right\} .
$$

Since we know that $-\frac{1}{\sqrt{s}} \log W(x, s)$ converges uniformly on $\bar{\Omega}$ to $d(x)$ as $s \rightarrow+\infty$, we can choose $s^{*}>0$ such that

$$
\left|-\frac{1}{\sqrt{s}} \log W(x, s)-d(x)\right|<\delta,
$$

for every $x \in \bar{\Omega}$ and every $s \geq s^{*}$. This latter inequality, together with (3.3), (3.4), and (3.5), gives, for every $s \geq s^{*}$, the following two estimates:

$$
\begin{aligned}
\int_{\partial B_{R}(0) \cap\left\{x_{N} \leq 0\right\}} x_{N} W(x, s) d S_{x} & \geq-\frac{1}{2} R e^{-4 \delta \sqrt{s}} \mathcal{H}^{N-1}\left(\partial B_{R}(0)\right), \\
\int_{V \cap \bar{\Omega}_{2 \delta}} x_{N} W(x, s) d S_{x} & \geq R \sin \theta e^{-3 \delta \sqrt{s}} \mathcal{H}^{N-1}\left(V \cap \bar{\Omega}_{2 \delta}\right) .
\end{aligned}
$$

Here $\mathcal{H}^{N-1}(\cdot)$ denotes the $(N-1)$-dimensional Hausdorff measure and $\Omega_{2 \delta}$ is defined by (2.10).

A consequence of (3.6) is that, for every $s \geq s^{*}$,

$$
\begin{aligned}
& \int_{\partial B_{R}(0)} x_{N} W(x, s) d S_{x} \\
& \quad \geq \int_{V \cap \bar{\Omega}_{2 \delta}} x_{N} W(x, s) d S_{x}+\int_{\partial B_{R}(0) \cap\left\{x_{N} \leq 0\right\}} x_{N} W(x, s) d S_{x} \\
& \quad \geq R e^{-3 \delta \sqrt{s}}\left[\sin \theta \mathcal{H}^{N-1}\left(V \cap \bar{\Omega}_{2 \delta}\right)-\frac{1}{2} e^{-\delta \sqrt{s}} \mathcal{H}^{N-1}\left(\partial B_{R}(0)\right)\right] .
\end{aligned}
$$

Therefore, we obtain a contradiction by observing that the first term of this chain of inequalities equals zero, by (3.1), while the last term can be made positive by choosing $s>0$ sufficiently large.

(iii), (iv), and (v). Let

$$
\Gamma=\left\{x \in \mathbb{R}^{N}: \operatorname{dist}(x, D)=R\right\} ;
$$


it is clear that $\Gamma \subset \partial \Omega$. Take any point $x \in \partial D$. Then, there exists a unique point $y \in \partial \Omega$ such that $\overline{B_{R}(x)} \cap \partial \Omega=\{y\}$. Indeed, since $\partial D$ is analytic by (ii), if $\tilde{y} \in \overline{B_{R}(x)} \cap \partial \Omega$ and $\tilde{y} \neq y$, then

$$
\frac{y-x}{|y-x|}=-\nu^{*}(x)=\frac{\tilde{y}-x}{|\tilde{y}-x|},
$$

where $\nu^{*}(x)$ is the interior unit normal vector to $\partial D$ at $x$, which is a contradiction. Since $\Omega$ enjoys the exterior sphere property, there exists a ball $B_{r}(z)$ such that $\overline{B_{r}(z)} \cap \bar{\Omega}=\{y\}$, and hence $\overline{B_{r}(z)} \cap \overline{B_{R}(x)}=\{y\}$. Therefore,

$$
\operatorname{dist}(z, D)=r+R \text { and } \overline{B_{r+R}(z)} \cap \bar{D}=\{x\} .
$$

Let $\kappa_{j}^{*}, j=1, \ldots, N-1$, denote the principal curvatures of the surface $\partial D$; (3.7) implies that

$$
\kappa_{j}^{*}(x) \geq-\frac{1}{r+R}, j=1, \ldots, N-1 .
$$

Since $\kappa_{j}^{*}>-\frac{1}{R}$ on $\partial D$, for every $j=1, \ldots, N-1, \Gamma$ is an analytic hypersurface diffeomorphic to $\partial D$ (see [G-T, Lemma 14.16]), and hence $\Gamma$ equals $\partial \Omega$. Assertions (iii), (iv), and (v) then follow at once.

(vi) Take any point $y \in \partial \Omega$. Assertions (iii) and (iv) imply that there exists a unique $x \in \partial D$ such that $\overline{B_{R}(y)} \cap \bar{D}=\{x\}$. Since $\partial D$ is analytic, $D$ satisfies the interior sphere condition, that is there exists a ball $B_{r}(z) \subset D$ such that $\overline{B_{r}(z)} \cap \partial D=\{x\}$. Therefore,

$$
d(z)=r+R \text { and } \overline{B_{r+R}(z)} \cap \partial \Omega=\{y\},
$$

and consequently

$$
\kappa_{j}(y) \leq \frac{1}{r+R}, j=1, \ldots, N-1 .
$$

Assertion (vi) is proved.

TheOREM 3.2. Let $\Omega$ be a bounded domain in $\mathbb{R}^{N}, N \geq 2$, satisfying the exterior sphere condition and suppose that $D$ is a domain satisfying the interior cone condition with boundary $\partial D$ and such that $\bar{D} \subset \Omega$.

Assume that the solution $u=u(x, t)$ to problem (1.1)-(1.3) satisfies condition (1.4).

Then, $\partial \Omega$ is analytic and (1.13) holds with $R$ given by (1.10). In particular, if $N=2, \Omega$ must be a ball.

Proof. First of all, by Lemma 3.1, both $\partial \Omega$ and $\partial D$ are analytic. Let $p$ and $q$ be two distinct points in $\partial \Omega$ and let

$$
P=p+R \nabla d(p), Q=q+R \nabla d(q) .
$$

Assertions (iv) and (v) from Lemma 3.1 guarantee that $P, Q \in \partial D$ and $P \neq Q$. (In fact, $p=y(P)$ and $q=y(Q)$ in (iv).) 
For $x \in B_{R}(0)$, consider the function

$$
v(x, t)=u(x+P, t)-u(x+Q, t) ;
$$

$v=v(x, t)$ satisfies the heat equation in $B_{R}(0) \times(0,+\infty)$ and by $(1.4)$

$$
v(0, t)=u(P, t)-u(Q, t)=0,
$$

for every $t>0$. Since $v$ is continuous up to $\partial B_{R}(0) \times(0,+\infty)$, by Theorem 2.1 we obtain

$$
\int_{\partial B_{R}(0)} v(x, t) d S_{x}=0
$$

for every $t>0$, and hence

$$
\int_{\partial B_{R}(P)} u(x, t) d S_{x}=\int_{\partial B_{R}(Q)} u(x, t) d S_{x}
$$

for every $t>0$. Therefore, in view of (1.5), we have

$$
\int_{\partial B_{R}(P)} W(x, s) d S_{x}=\int_{\partial B_{R}(Q)} W(x, s) d S_{x}
$$

for every $s>0$. Assertions (v) and (vi) from Lemma 3.1 make sure that we can apply Theorem 2.3 (with $\varphi=1$ ) to (3.11). We multiply both sides of (3.11) by $s^{\frac{N-1}{4}}$ and take the limits as $s \rightarrow+\infty$. Since $\partial B_{R}(P) \cap \partial \Omega=\{p\}$ and $\partial B_{R}(Q) \cap \partial \Omega=\{q\}$, after some manipulation, we obtain:

$$
\prod_{j=1}^{N-1}\left[\frac{1}{R}-\kappa_{j}(p)\right]=\prod_{j=1}^{N-1}\left[\frac{1}{R}-\kappa_{j}(q)\right],
$$

that is (1.13) holds.

We quote A. D. Aleksandrov's uniqueness theorem from [Alek, p. 412], adjusted to our notations. A special case of this theorem is the well-known Soap-Bubble Theorem (see also $[\mathrm{R}]$ ).

TheOREM 3.3 (Aleksandrov). Let $\Phi=\Phi\left(\kappa_{1}, \cdots, \kappa_{N-1}\right)$ be a continuously differentiable function, defined for $\kappa_{1} \geq \cdots \geq \kappa_{N-1}$, and subject to the condition $\frac{\partial \Phi}{\partial \kappa_{i}}>0(i=1, \cdots, N-1)$.

Suppose that in $\mathbb{R}^{N}$ we have a twice-differentiable closed surface $S$ without self-intersections and with bounded principal curvatures.

If on the surface $S$ the function $\Phi$ of its principal curvatures $\kappa_{1}, \cdots, \kappa_{N-1}$ has at all points one and the same value, then $S$ is a sphere.

Proof of Theorem 1.1. By Theorem 3.2, it suffices to consider the case where $N \geq 3$. 
We set

$$
\Phi=\Phi\left(\kappa_{1}, \cdots, \kappa_{N-1}\right)=-\prod_{j=1}^{N-1}\left[\frac{1}{R}-\kappa_{j}\right]
$$

and observe that

$$
\frac{\partial \Phi}{\partial \kappa_{i}}>0 \quad(i=1, \cdots, N-1), \text { if } \max _{1 \leq j \leq N-1} \kappa_{j}<\frac{1}{R} .
$$

Since condition (1.13) holds by Theorem 3.2, we infer that the function $\Phi$ is constant on $\partial \Omega$.

Therefore, by applying Theorem 3.3 to each connected component of $\partial \Omega$, we conclude that $\partial \Omega$ must be a sphere.

Remark. The method of proof of Theorem 3.3 is called Aleksandrov's reflection principle or the method of moving planes, which is based on the maximum principle for elliptic partial differential equations of second order.

In fact, by using local coordinates, the condition $\Phi\left(\kappa_{1}, \ldots, \kappa_{N-1}\right)=$ constant on the surface $S$ can be converted into a second order partial differential equation which is of elliptic type, since $\frac{\partial \Phi}{\partial \kappa_{i}}>0(i=1, \cdots, N-1)$. In the case the function $\Phi$ is given by (3.12), we obtain an equation of Monge-Ampère type.

Acknowledgement. The authors would like to thank Professor Toshio Mikami for the reference $[\mathrm{F}-\mathrm{W}]$ on the asymptotic behavior as $t \rightarrow 0$ of the solution of problem (1.1)-(1.3) and Professor M. Bardi for the references [E-I] and [Va].

Università di Firenze, Firenze, Italy

E-mail address: magnanin@math.unifi.it

EHIME UNIVERSITY, EHIME, JAPAN

E-mail address: sakaguch@dpc.ehime-u.ac.jp

\section{ReFERENCES}

[Alek] A. D. Aleksandrov, Uniqueness theorems for surfaces in the large V, Vestnik Leningrad Univ. 13 (1958), 5-8 (English translation: Trans. Amer. Math. Soc. 21 (1962), 412-415).

[A1] G. Alessandrini, Matzoh ball soup: a symmetry result for the heat equation, $J$. Analyse Math. 54 (1990), 229-236.

[A2] Characterizing spheres by functional relations on solutions of elliptic and parabolic equations, Applicable Anal. 40 (1991), 251-261.

[B-L] H. L. Brascamp and E. H. Lieb, On extensions of Brunn-Minkowski and PrékopaLeindler theorems, including inequalities for log-concave functions, and with an application to the diffusion equation, J. Funct. Anal. 22 (1976), 366-389.

[deB] N. G. De Bruisn, Asymptotic Methods in Analysis, Bibliotheca Mathematica 4, North-Holland Publ. Co., Amsterdam; P. Noordhoff Ltd., Groningen; Interscience Publ. Inc., New York, 1958. 
[Ev] L. C. Evans, Partial Differential Equations, Amer. Math. Soc., Providence, RI, 1998.

[E-I] L. C. Evans and H. Ishit, A PDE approach to some asymptotic problems concerning random differential equations with small noise intensities, Ann. Inst. Henri Poincaré 2 (1985), 1-20.

[F-W] M. I. Freidlin and A. D. Wentzell, Random Perturbations of Dynamical Systems, Springer-Verlag, New York, 1984.

[G-H-L] S. Gallot, D. Hulin, and J. Lafontaine, Riemannian Geometry (Second Edition), Springer-Verlag, New York, 1993.

[G-T] D. Gilbarg and N. S. Trudinger, Elliptic Partial Differential Equations of Second Order (Second Edition), Springer-Verlag, New York, 1983.

[Kl] M. S. Klamkin, A physical characterization of a sphere (Problem $64-5^{*}$ ), SIAM Review 6 (1964), 61.

[Ko] N. J. KorevaAr, Convex solutions to nonlinear elliptic and parabolic boundary value problems, Indiana Univ. Math. J. 32 (1983), 603-614.

[LC] T. Levi-Civita, Famiglie di superficie isoparametriche nell'ordinario spazio euclideo, Atti Accad. Naz. Lincei Rend. Cl. Sci. Fis. Mat. Natur. 26 (1937), 355-362.

[M-S1] R. Magnanini and S. Sakaguchi, The spatial critical points not moving along the heat flow, J. Analyse Math. 71 (1997), 237-261.

[M-S2] The spatial critical points not moving along the heat flow II: The centrosymmetric case, Math. Z. 230 (1999), 695-712, Corrigendum, ibid. 232 (1999), 389.

[M-S3] — On heat conductors with a stationary hot spot, Ann. Mat. Pura Appl., to appear.

[R] R. C. Reilly, Mean curvature, the Laplacian, and soap bubbles, Amer. Math. Monthly 89 (1982), 180-188, 197-198.

[R-R] I. S. Rombauer and M. Rombauer Becker, Joy of Cooking, Plume, New American Library, New York, 1973.

[Sa1] S. SAKAGUCHI, When are the spatial level surfaces of solutions of diffusion equations invariant with respect to the time variable?, J. Analyse Math. 78 (1999), 219-243.

[Sa2] Stationary critical points of the heat flow in spaces of constant curvature, J. London Math. Soc. 63 (2001), 400-412.

[Seg] B. Segre, Famiglie di ipersuperficie isoparametriche negli spazi euclidei ad un qualunque numero di dimensioni, Atti Accad. Naz. Lincei Rend. Cl. Sci. Fis. Mat. Natur. 27 (1938), 203-207.

[Ser] J. Serrin, A symmetry problem in potential theory, Arch. Rational Mech. Anal. 43 (1971), 304-318.

[Va] S. R. S. VARADHAN, On the behavior of the fundamental solution of the heat equation with variable coefficients, Comm. Pure Appl. Math. 20 (1967), 431-455.

[Z] L. Zalcman, Some inverse problems of potential theory, Contemp. Math. 63 (1987), $337-350$.

(Received April 11, 2001) 